\title{
ACCESO A LA INFORMACIÓN PÚBLICA Y SU REUTILIZACIÓN EN LAS COMUNIDADES AUTÓNOMAS: EVALUACIÓN DE LA REUTILIZACIÓN DE DATOS ABIERTOS
}

\author{
Access to and reuse of public information in Spanish \\ regions: evaluation of open data reuse
}

Adrián Vicente-Paños y Aurea Jordán-Alfonso

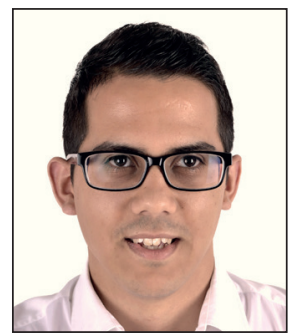

Adrián Vicente-Paños es graduado en Gestión y Administración pública por la Universitat Politècnica de València y Máster en Gestión de Empresas, Productos y Servicios por la misma universidad. Desarrolla su actividad profesional en el Servicio de Transparencia de la Diputació de València. Sus líneas de investigación se centran en la gestión de la información y la gestión de la innovación en organizaciones públicas.

http://orcid.org/0000-0003-2639-5701

Diputació de València, Servicio de Transparencia Hugo de Moncada, 9. 46010 Valencia, España advipao@gmail.com

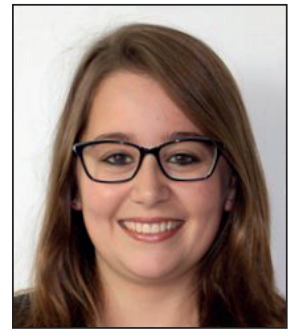

Aurea Jordán-Alfonso es graduada en Ingeniería Química por la Universitat Politècnica de València y Máster en Gestión de Empresas, Productos y Servicios por la misma universidad. Desarrolla su actividad profesional en el Centro de Investigación en Economía y Gestión de la Salud, cuyas principales líneas de trabajo son la farmacoeconomía y la innovación.

http://orcid.org/0000-0002-2694-3897

Universitat Politècnica de València, Centro de Ingeniería Económica Camino de Vera, s/n. 46022 Valencia, España aujoral@gmail.com

\section{Resumen}

Se analiza la situación en materia de acceso a la información del sector público y su reutilización en las comunidades autónomas de España. Para ello se utiliza Meloda, métrica para evaluar la reutilización de datos publicados en los portales de datos abiertos. Además, se estudia la correlación del número de conjuntos de datos disponibles en los portales de datos abiertos, el Índice de Transparencia Internacional de las comunidades autónomas y la media de Meloda para cada comunidad autónoma. Se han estudiado 2.165 conjuntos de datos. El 95,15\% de los datos publicados en el Estado Español por comunidades autónomas en portales de datos abiertos son de reutilización avanzada o de reutilización avanzada con alguna característica mejorable. Las comunidades que están liderando este proceso son Aragón, Cataluña, Comunidad Foral de Navarra y País Vasco.

\section{Palabras clave}

Datos abiertos; Información pública; Conjuntos de datos; Reutilización; Transparencia; MEtric for reLeasing Open DAta; Meloda.

\begin{abstract}
The situation of access to public sector information and its reuse in Spanish regions is analyzed. For this purpose, it was used Meloda, a metric to assess open data reuse published on open data portals. Furthermore, the correlation between the number of datasets available on open data portals, the International Transparency Index of Spanish regions, and the Meloda average for each Spanish region are studied. 2,165 datasets have been examined. 95.15\% of data published by Spanish regions on open data portals is optimum for reuse, or suitable for reuse but with some improvement needed. The public bodies which are leading this process are those of Aragón, Cataluña, Comunidad Foral de Navarra and País Vasco.
\end{abstract}

\section{Keywords}

Open data; Datasets; Public information; Information reuse; Transparency; MEtric for reLeasing Open DAta; Meloda. 
Vicente-Paños, Adrián; Jordán-Alfonso, Aurea (2017). "Acceso a la información pública y su reutilización en las comunidades autónomas: evaluación de la reutilización de datos abiertos". El profesional de la información, v. 26, n. 3, pp. 381-391.

https://doi.org/10.3145/epi.2017.may.04

\section{Introducción: transparencia por diseño y acceso a la información pública}

La transparencia en todas sus dimensiones: activa $^{1}$, pasiva $^{2}$ y colaborativa ${ }^{3}$ (Villoria; Cruz-Rubio, 2015), el acceso a la información pública a través de datos abiertos (open data) y la reutilización de la información del sector público (RISP), requieren abandonar la autonomía institucional en el cumplimiento de objetivos generales para impulsar un modelo sostenible e integral de administración electrónica que contemple un verdadero marco de interoperabilidad (García-González, 2016). Este marco debe atender de forma integrada a los tres ámbitos en los que se articulan las organizaciones públicas: sociotécnico, político-cultural y de control y mejora (Ramió, 2010).

A partir del preámbulo de la Ley 19/2013, de 9 de diciembre, de transparencia, acceso a la información pública y buen gobierno se puede identificar la necesidad de una transparencia por diseño ${ }^{4}$.

"la transparencia, el acceso a la información pública y las normas de buen gobierno deben ser los ejes fundamentales de toda acción política" (España, 2013).

En esta perspectiva, la transparencia y los datos abiertos se deberían abordar desde un ejercicio profundo de planificación de los procesos de gestión de la información que se dan en todos los niveles de las organizaciones.

Además, se da la posibilidad de explorar las interacciones entre información del sector público, datos gubernamentales abiertos, datos abiertos y datos masivos mediante técnicas y métodos de gestión de la información como la minería de datos, el análisis del sentimiento o el modelado de políticas (Ferrer-Sapena; Sánchez-Pérez, 2013; Ubaldi, 2013; Arenilla, 2014).

Una vez identificado el valor de la transparencia por diseño se requiere dirigir todos los esfuerzos de gestión de la información hacia una transparencia abierta ${ }^{5}$, es decir que la publicidad activa asuma los valores de los datos abiertos:

"disponibilidad íntegra de acceso, posibilidad de redistribución, y licencia para la reutilización" (Ferrer-Sapena; Peset; Aleixandre-Benavent, 2011),

Se debe entender la transparencia de manera disruptiva, es decir datos nuevos o radicalmente mejorados que favorecen la provisión de servicios inexistentes en la actualidad, y reactiva, a partir del ejercicio del derecho de acceso a la información pública.

Como se recoge en Vicente-Paños (2016), a partir del estudio Creating value through open data de la Comisión Europea (Carrara et al., 2015), estos datos abiertos, sustentados en los sistemas de e-administración, presentarían un poten- cial valor económico estimado en 325.000 millones de euros en el período 2016-2020, el tamaño del mercado directo acumulativo para la Unión Europea junto con Liechtenstein, Noruega y Suiza, UE28+ (Vicente-Paños, 2016).

Se pueden destacar algunas iniciativas que monitorizan los datos abiertos:

- Open Data Index (2015). Primera iniciativa global del seguimiento de los datos abiertos de la Administración promovida por la Open Knowledge Foundation International en la que se identifica que el $9 \%$ de los conjuntos de datos clave del índice que analiza 122 países, están abiertos. http://index.okfn.org

- Open Data Barometer (2015). Impulsado por la World Wide Web Foundation, cubre 92 países clasificándolos según su nivel de preparación, implementación e impacto de los datos abiertos en los negocios, la política y la sociedad civil. En el caso español la puntuación obtenida es de 78 puntos en preparación, 57 en implementación y 63 en impacto.

http://opendatabarometer.org

- Open Data Monitor (2015). Se centra en los siguientes elementos (resultados aplicados al caso español): licencias abiertas, 78\%; legible por máquina, 49\%; disponibilidad, $57 \%$; completitud de los metadatos, $58 \%$; la puntuación global de calidad es del $60 \%$ en España.

http://opendatamonitor.eu

Como antecedente a estas iniciativas se puede identificar la plataforma ePSI Scoreboard (2013) que de acuerdo con la Directiva 2003/98/CE sobre reutilización de la información del sector público (Comisión Europea, 2003), modificada por la Directiva 2013/37/UE del Parlamento Europeo y del Consejo, de 26 de junio de 2013 (Comisión Europea, 2013), contemplaba en el índice los siguientes aspectos:

- situación del país en relación a la trasposición de la Directiva 2003/98/CE, puesta en práctica de las políticas de reutilización;

- formatos en los que se dispone la información pública;

- tarificación;

- medidas para el control de acuerdos exclusivos;

- disponibilidad de catálogos locales y regionales de datos abiertos;

- celebración de eventos y actividades sobre información del sector público.

En el contexto español se identifican 15 iniciativas sobre datos abiertos a nivel autonómico, 92 en la administración local, 34 en la administración del Estado y 10 en universidades (datos.gob.es, 2016). En la Unión Europea 24 países han implementado un portal de datos abiertos nacional con una media de 5.035 conjuntos de datos por país, por 
lo que la evolución de los datos abiertos en esos países se encuentra en una fase inicial (Vicente-Paños, 2016). Diez de estos países (el 32\%) configuran el grupo destacado de "fijadores de tendencias" (trend setters) del sector de datos abiertos en Europa (Carrara et al., 2015). Los países que están liderando este proceso en la Unión Europea son España, Francia, Irlanda, Austria y Reino Unido (European Data Portal, 2017).

Los principales objetivos de este trabajo son:

- identificar la situación del acceso a la información del sector público y su reutilización en las comunidades autónomas de España;

- realizar un análisis, documentar y probar una métrica para evaluar la reutilización de datos abiertos en las comunidades autónomas.

\section{Metodología}

Para obtener una visión global sobre el acceso a la información del sector público y su reutilización se siguen tres etapas:

- preparación;

- definición del tamaño de la muestra para un nivel de confianza del 95\%, e = 5\%;

- clasificación de los conjuntos de datos según su categoría, creación de un repositorio de datos, análisis mediante Meloda versión 4.08 y confirmación de su consistencia.

Este análisis se ha realizado entre el 16 de julio y el 19 de agosto de 2016.

\subsection{Preparación}

Para identificar el número de conjuntos de datos que se evaluarán con la métrica Meloda se analizan los portales de datos abiertos de las comunidades autónomas atendiendo a las siguientes características:

- organización: se identifican los portales de las 17 comunidades autónomas del Estado español;

- portal de datos abiertos: se diferencia entre portal de transparencia y portal de datos abiertos;

- reutilización de la información pública: se analiza el número de conjuntos de datos publicados por portales de datos abiertos para extraer una muestra representativa. Para realizar este análisis se crea una base de datos en

Tabla 1. Ficha técnica

\begin{tabular}{|l|l|}
\hline \multicolumn{2}{|c|}{ Ficha técnica } \\
\hline Área & Comunidades autónomas, España \\
\hline Universo & $\begin{array}{l}\text { Conjuntos de datos disponibles en los portales } \\
\text { de datos abiertos }\end{array}$ \\
\hline Tamaño muestral & 2.165 conjuntos de datos \\
\hline Error muestral & $\begin{array}{l} \pm 5 \% \\
(\mathrm{p}=\mathrm{q}=0,5)\end{array}$ \\
\hline Nivel de confianza & $95 \%(\mathrm{Z}=1,96)$ \\
\hline Muestreo & Aleatorio estratificado proporcional \\
\hline Trabajo de campo & Agosto de 2016 \\
\hline
\end{tabular}

Excel; los conjuntos de datos se seleccionan de forma aleatoria combinando su descarga manual con su visualización online.

\subsection{Selección de la muestra}

Para la realización de este estudio se recogen los 7.098 conjuntos de datos publicados en los portales de datos abiertos de las 17 comunidades autónomas del Estado español. A continuación se analizan mediante Meloda, métrica para evaluar la reutilización de datos abiertos. Para ello se extrae una muestra representativa, para un nivel de confianza del 95\%, y $p=q$, el error muestral es de $\pm 5 \%$ para el conjunto de la muestra de los datos a estudiar en cada comunidad (tablas 1 y 2 ).

Tabla 2. Número de datos publicados y analizados mediante la metodología Meloda en cada comunidad autónoma

\begin{tabular}{|l|r|r|}
\hline \multicolumn{1}{|c|}{ Comunidad autónoma } & $\begin{array}{c}\text { N. datos } \\
\text { publicados }\end{array}$ & $\begin{array}{c}\text { N. datos } \\
\text { analizados }\end{array}$ \\
\hline Andalucía & 190 & 127 \\
\hline Aragón & 2.659 & 336 \\
\hline Canarias & 45 & 40 \\
\hline Cantabria & 341 & 181 \\
\hline Castilla y León & 201 & 132 \\
\hline Castilla-La Mancha & 15 & 14 \\
\hline Catalunya & 1.490 & 306 \\
\hline Comunidad de Madrid & 0 & 0 \\
\hline Comunidad Foral de Navarra & 744 & 254 \\
\hline Comunitat Valenciana & 279 & 162 \\
\hline Extremadura & 25 & 24 \\
\hline Galicia & 332 & 178 \\
\hline Illes Balears & 42 & 38 \\
\hline La Rioja & 153 & 225 \\
\hline País Vasco & 540 & 38 \\
\hline Principado de Asturias & 42 & \\
\hline Región de Murcia & 098 & 165 \\
\hline Total & & 225 \\
\hline
\end{tabular}




\subsection{Meloda}

A diferencia de otras métricas orientadas a evaluar datos enlazados y la calidad y la arquitectura de los datos (Berners-Lee, 2009; Pipino; Lee; Wang, 2002; Ren; Glissmann, 2012), Meloda permite evaluar la reutilización de datos abiertos aportando una visión global sobre sus oportunidades de reutilización (Abella; Ortiz-de-Urbina-Criado; DePablos-Heredero, 2014). Permite, además, identificar la situación del acceso a la información del sector público y su reutilización en las comunidades autónomas, así como estrategias futuras de publicación a los gestores de información. Esta métrica no ha sido utilizada en una muestra representativa de conjuntos de datos de comunidades autónomas como la descrita en el apartado 2.2.

La métrica recoge en su versión 4.08 las variables información geolocalizada e información en tiempo real. Esta metodología en sí misma no evalúa los portales de datos abiertos completos, sino que centra el objeto de su análisis en los conjuntos de datos de forma individual. A partir del análisis de sus seis dimensiones se pretende obtener una visión integrada de la situación de los datos abiertos en las comunidades autónomas basada en los elementos comunes detectados en los portales. A partir de Abella (2016), las seis dimensiones que se evalúan son (tabla 3):

- estándares técnicos: estructura técnica en que se suministra el dato;

- acceso: mecanismo por el cual se hace posible la conexión con la información o su descarga;

- legal: licencia que se asigna al conjunto de datos;

- modelo de datos: tipo de dato utilizado por el publicador y capacidad de compartir la información publicada con otras entidades generando un estándar común;

- información geolocalizada: datos publicados con algunos campos que identifican la ubicación de los datos;

- información en tiempo real: actualización de los datos publicados de manera periódica.

Una vez asignados los porcentajes de las seis dimensiones para un conjunto de datos, se calcula la raíz octava de su producto y se multiplica por 100 para obtener un número que varía entre 0 y 100. La siguiente fórmula se aplica a cada conjunto de datos de manera individual:

Meloda $=100 \cdot \sqrt{ }(\sqrt{ }(\mathrm{V}($ Estándares técnicos $\cdot$ Acceso $\cdot$ Legal · Modelo de datos · Información geolocalizada . Información en tiempo real)))

Tabla 3. Meloda: dimensiones y niveles

\begin{tabular}{|c|c|c|c|c|c|}
\hline \multicolumn{2}{|l|}{ Estándares técnicos } & \multicolumn{2}{|l|}{ Acceso } & \multicolumn{2}{|l|}{ Legal } \\
\hline Nivel & Peso (\%) & Nivel & Peso (\%) & Nivel & Peso (\%) \\
\hline Estándar privativo no reutilizable & 10 & Sin acceso & 0 & Copyright & 0 \\
\hline Estándar privativo reutilizable & 35 & Acceso vía web con registro & 10 & Uso privado & 10 \\
\hline Estándar abierto & 60 & Acceso directo vía web & 50 & Uso no comercial & 25 \\
\hline \multirow[t]{2}{*}{ Estándar abierto con metadatos } & 100 & Acceso vía web con parámetros & 90 & Uso comercial & 90 \\
\hline & & Acceso completo (API) & 100 & Uso no limitado con autoría & 100 \\
\hline \multicolumn{2}{|l|}{ Modelo de datos } & \multicolumn{2}{|c|}{ Información geolocalizada } & \multicolumn{2}{|c|}{ Información en tiempo real } \\
\hline Nivel & Peso (\%) & Nivel & Peso (\%) & Nivel & Peso (\%) \\
\hline Sin modelo publicado & 15 & No hay información geográfica & 15 & Mayor que una semana & 15 \\
\hline Modelo con campos de datos & 35 & Campo de texto simple & 30 & Días & 40 \\
\hline Modelo con especificaciones de campos & 50 & Campo de texto complejo & 50 & Horas & 70 \\
\hline Modelo externo normalizado & 90 & Coordenadas & 90 & Minutos & 90 \\
\hline Modelo externo y generalizado & 100 & Información geográfica completa & 100 & Segundos & 100 \\
\hline
\end{tabular}

Basado en: Abella; Ortiz-de-Urbina-Criado; De-Pablos-Heredero, 2014; Abella, 2016. 
El resultado se clasifica, dependiendo del rango al que pertenece, en una de las cuatro categorías disponibles (tabla 4). La clasificación de los datos abiertos indicará el grado de adecuación de un conjunto de datos para su reutilización.

\section{4. Índice de Transparencia Internacional}

Se comparan los resultados del análisis de los conjuntos de datos con Meloda, con la puntuación que Transparencia Internacional dio a las comunidades autónomas en 2014. El índice de Transparencia Internacional España, Índice de las Comunidades Autónomas (Transparencia Internacional España, 2014), cuenta con 80 indicadores divididos en 6 áreas:

- información sobre la comunidad autónoma (21);

- relaciones con los ciudadanos y la sociedad (14);

- transparencia económico-financiera (13);

- transparencia en las contrataciones de servicios, obras y suministros (9);

- transparencia sobre ordenación del territorio, urbanismo y obras públicas (7);

- indicadores nueva Ley de transparencia (16).

El $88,24 \%$ de las comunidades autónomas ha implementado un portal de datos abiertos

El Índice de Transparencia de las Comunidades Autónomas (Incau) persigue un doble objetivo:

"por una parte, realizar una evaluación del nivel de transparencia de los gobiernos de las comunidades autónomas, y por otra, impulsar y propiciar el aumento de la información que estas instituciones ofrecen a los ciudadanos y a la sociedad en su conjunto" (Transparencia Internacional España, 2016).

Esta evaluación se basa en un cuestionario que contiene los citados indicadores, y que está previamente cumplimentado por Transparencia Internacional España. A la puntuación mínima inicial otorgada por esta organización, las comunidades autónomas pueden dar su conformidad o añadir a su web o portal de transparencia información adicional que será indicada en el cuestionario para su validación por Transparencia Internacional.

\section{Resultados}

Las comunidades que más datos abiertos han publicado son (gráfico 1):

- Aragón (2.659);

- Catalunya (1.490);
- Comunidad Foral de Navarra (744);

- País Vasco (540).

Mientras que las comunidades que menos datos han publicado son:

- Castilla-La Mancha (15);

- Extremadura (25);

- Principado de Asturias (42);

- Illes Balears (42).

La Comunidad de Madrid y la Región de Murcia, ambas uniprovinciales, no tienen portal de datos abiertos, por lo que el número de conjuntos de datos publicados es cero. En cambio, estas instituciones autonómicas sí que tienen un portal de transparencia. Dependiendo de la comunidad autónoma la información se publica en varias webs como: portal de transparencia, portal de datos abiertos o web institucional.

La Región de Murcia publicó en su web que durante 2014 lanzaría su portal de datos abiertos (Vicente-Paños, 2015), portal que en enero de 2017 no está operativo. Esta comunidad autónoma cuenta con la Estrategia Regional de Datos Abiertos aprobada en Consejo de Gobierno de 1 de junio de 2016. En contraste con la situación de la Comunidad de Madrid, en Murcia la Consejería de Presidencia tiene atribuidas las funciones de transparencia y buen gobierno. Además, cuentan con una Oficina de la Transparencia y la Participación Ciudadana.

Observando los conjuntos de datos publicados en portales de datos abiertos, el 37,46\% de los publicados en España

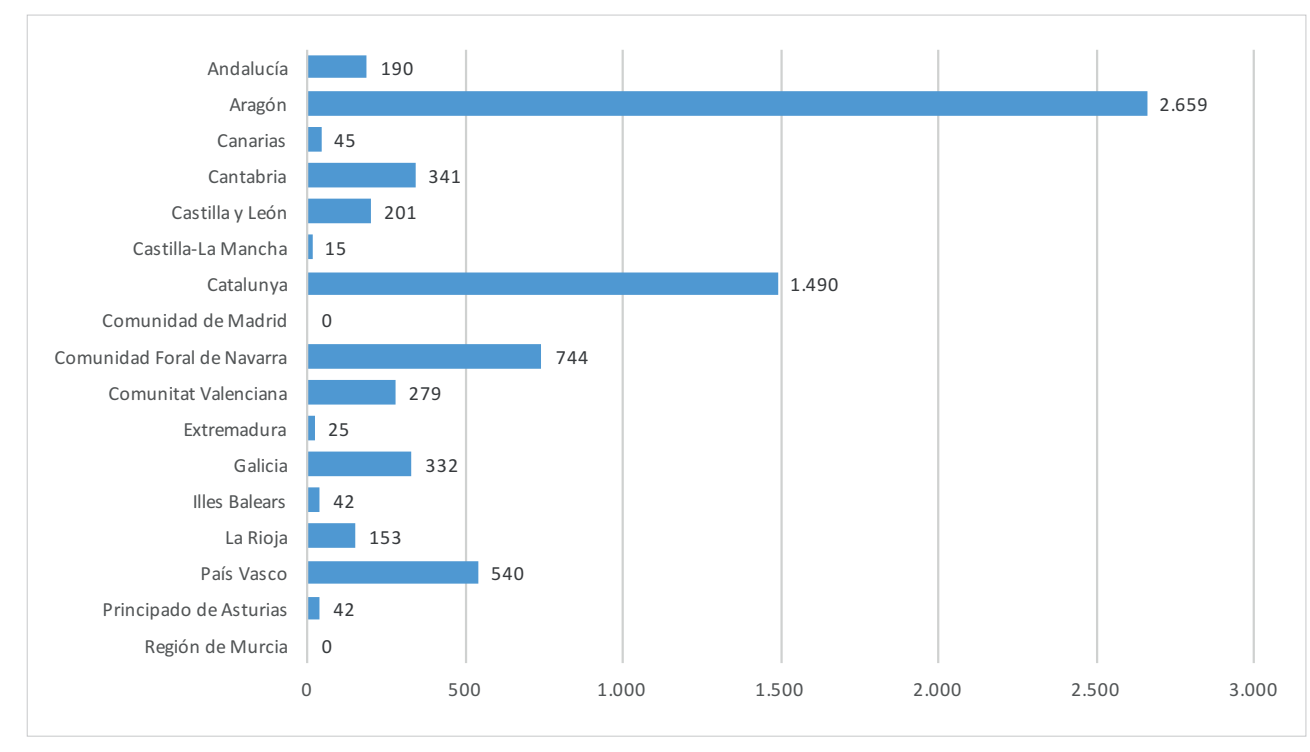

Gráfico 1. Número de conjuntos de datos publicados por comunidad autónoma 


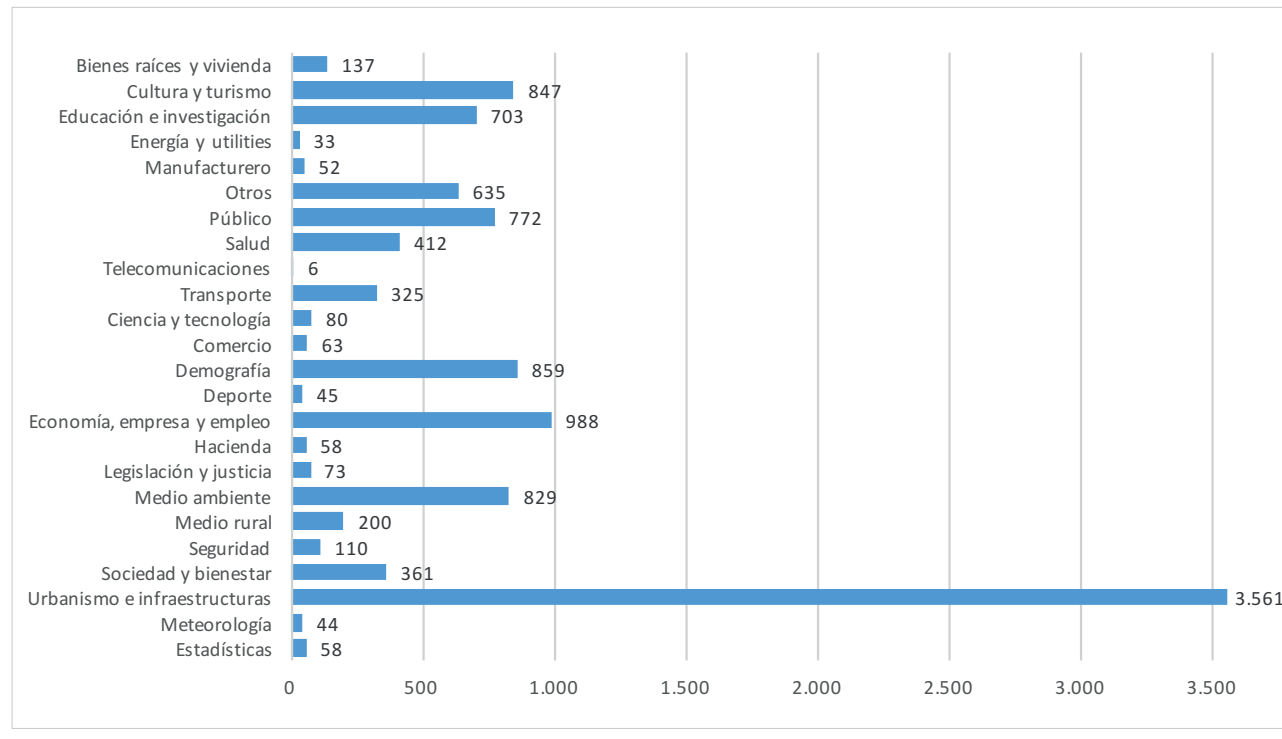

Gráfico 2. Número de conjuntos de datos publicados por categoría avanzada con alguna característica mejorable, a excepción de la media de los datos del Principado de Asturias $(45,59)$ que pertenece a la categoría 2 , reutilización básica (tabla 5).

La distribución por categorías es aplicable a un conjunto de datos de manera individual, pero se destaca la categoría a la que pertenecería un supuesto conjunto de datos promedio de cada comunidad autónoma para tener una visión aproximada del tipo de datos abiertos publicados en cada portal. pertenecen a Aragón, el 20,99\% a Catalunya, el 10,48\% a Navarra y el $7,61 \%$ al País Vasco. Las otras 13 comunidades reúnen el $23,46 \%$ restante.

La categoría en la que más datos abiertos se publican es en la de urbanismo e infraestructuras

Para facilitar su búsqueda, los 7.098 conjuntos de datos se clasifican en sus respectivos portales en más de una categoría de las 24 identificadas y el sumatorio de datos abiertos es de 11.251. La categoría en la que se publican más datos abiertos es urbanismo e infraestructuras, con 3.561 conjuntos de datos, mientras que en todas las demás categorías hay menos de mil (gráfico 2).

El análisis de una muestra representativa de conjuntos de datos mediante $\mathrm{Me}$ loda señala que Canarias tiene los datos con la media más elevada $(84,06)$, es decir, tienen mayor calidad para su reutilización y, por tanto, de reutilización avanzada (categoría 4). La media de los datos de Catalunya $(79,59)$ y Aragón $(78,94)$ también es de categoría 4 por ser mayor de 75. Las medias que obtienen el resto de datos por comunidad autónoma pertenecen a la categoría 3, lo que indica reutilización
Tabla 5. Análisis comparativo de resultados

\begin{tabular}{|l|c|c|c|c|}
\hline \multicolumn{1}{|c|}{ Comunidad autónoma } & Media Meloda & Moda Meloda & Mínimo Meloda & Máximo Meloda \\
\hline Andalucía & 55,06 & 56,87 & 34,09 & 75,83 \\
\hline Aragón & 78,94 & 78,89 & 78,89 & 95,64 \\
\hline Canarias & 84,06 & 76,84 & 76,84 & 97,40 \\
\hline Cantabria & 65,88 & 62,23 & $0,00^{*}$ & 75,45 \\
\hline Castilla y León & 63,97 & 56,87 & $0,00^{*}$ & 76,84 \\
\hline Castilla-La Mancha & 57,84 & 61,42 & 42,80 & 61,42 \\
\hline Catalunya & 79,59 & 76,84 & 72,08 & 97,40 \\
\hline Comunidad de Madrid & 0,00 & 0,00 & 0,00 & 0,00 \\
\hline Comunidad Foral de Navarra & 55,68 & 53,16 & $0,00^{*}$ & 85,42 \\
\hline Comunitat Valenciana & 71,18 & 73,49 & 58,38 & 77,86 \\
\hline Extremadura & 57,62 & 57,62 & 57,62 & 57,62 \\
\hline Galicia & 61,88 & 56,87 & 45,46 & 97,40 \\
\hline Illes Balears & 63,37 & 60,62 & 60,62 & 73,49 \\
\hline La Rioja & 63,05 & 60,62 & 60,62 & 76,84 \\
\hline País Vasco & 69,51 & 56,87 & $0,00^{*}$ & 94,39 \\
\hline Principado de Asturias & 45,59 & 45,02 & 42,24 & 50,53 \\
\hline Región de Murcia & 0,00 & 0,00 & 0,00 & 0,00 \\
\hline
\end{tabular}

*Los conjuntos de datos indicados no tienen recursos o muestran un error de visualización.
El valor que más veces se ha obtenido en la comunidad autónoma se muestra en la columna Moda de la tabla 5. Aragón tiene la mayor moda $(78,89)$, por lo que cabe destacar que aunque su media no es la mejor $(78,94)$, la desviación típica de la muestra es pequeña $(0,91)$, es decir, publica unos datos con características muy similares y de reutilización avanzada.

Las muestras de Extremadura y el Principado de Asturias también son homogéneas puesto que sus desviaciones típicas son 0 y 1,86 respectivamente. El resto de muestras tienen una desviación típica mayor que varía entre 4,22 y 17,80 , por lo que los datos de una misma comunidad autónoma obtienen una puntuación de Meloda muy heterogénea entre sí. 
Tras el análisis individual de cada conjunto de datos con Meloda y su categorización, se obtiene que la categoría 3 es la que contiene más datos abiertos con un $55,84 \%$ de los datos, seguida de la 4 con un $39,31 \%$. La categoría 2 tiene el $4,06 \%$ de los datos abiertos y la categoría 1 el $0,79 \%$. Por tanto, la mayoría de los datos abiertos publicados pertenecen a la categoría 3 , que indica una reutilización avanzada con alguna característica mejorable, y a la 4 , reutilización avanzada. El porcentaje de datos que pertenecen a las categorías 1

y 2 es del 4,85\%, lo que indica que el número de conjuntos de datos no adecuados para su reutilización es muy reducido.

La mayoría de los datos publicados en España en portales de datos abiertos son de reutilización avanzada con alguna característica mejorable

El porcentaje por categoría refleja que sólo Aragón y Canarias tienen el $100 \%$ de sus datos abiertos de reutilización avanzada, seguido de Catalunya con un 98,69\%. Once comunidades tienen la mayoría de sus conjuntos de datos clasificados como categoría 3, reutilización avanzada con alguna característica mejorable: Andalucía (59,84\%), Cantabria (60,77\%), Castilla y León (98,48\%), Castilla-La Mancha (92,86\%), Navarra $(97,24 \%)$, Comunitat Valenciana $(98,77 \%)$, Extremadura (100\%), Galicia (86,52\%), Illes Balears (100\%), La Rioja $(92,73 \%)$ y País Vasco $(65,78 \%)$. El Principado de Asturias tiene el 92,11\% de categoría 2, reutilización básica (gráfico 3).

El análisis de correlación entre la puntuación obtenida por las comunidades autónomas en el Índice de Transparencia Internacional (2014), el número de conjuntos de datos publicados y la media Meloda obtenida (tabla 6), da como resultado una correlación positiva $(0,1876)$ entre la puntuación dada por Transparencia Internacional a las comunidades autónomas y el número de conjuntos de datos publicados, es decir, a mayor número de datos abiertos publicados mayor es la puntuación obtenida en el Índice de Transparencia Internacional. Esta correlación no tiene un valor muy elevado, el valor 1 indica que la correlación es al 100\% mientras que el valor 0 indica que no hay correlación.

Además, el índice de correlación entre el número de datos publicados y su grado de reutilización atendiendo a los resultados obtenidos con Meloda es de 0,4132, es decir, que hay una correlación mayor que en el caso anterior $y$, por tanto, es más probable que a un mayor número de conjuntos de datos publicados en los portales de datos abiertos, la media de los valores obtenidos con Meloda sea mayor.

Por último, atendiendo a la correlación entre la puntua-

Tabla 6. Resultados Índice de Transparencia Internacional, número de datos publicados y media Meloda para cada comunidad autónoma

\begin{tabular}{|l|c|c|c|}
\hline \multicolumn{1}{|c|}{ Comunidad autónoma } & $\begin{array}{c}\text { Transparencia } \\
\text { Internacional }\end{array}$ & $\begin{array}{c}\text { N. datos } \\
\text { publicados }\end{array}$ & Media Meloda \\
\hline Andalucía & 88 & 190 & 55,06 \\
\hline Aragón & 85 & 2.659 & 78,94 \\
\hline Canarias & 80 & 45 & 84,06 \\
\hline Cantabria & 88 & 341 & 65,88 \\
\hline Castilla y León & 100 & 201 & 63,97 \\
\hline Castilla-La Mancha & 84 & 15 & 57,84 \\
\hline Catalunya & 100 & 1.490 & 79,59 \\
\hline Comunidad de Madrid & 65 & 0 & 0,00 \\
\hline Comunidad Foral de Navarra & 89 & 744 & 55,68 \\
\hline Comunitat Valenciana & 93 & 279 & 71,18 \\
\hline Extremadura & 85 & 25 & 57,62 \\
\hline Galicia & 94 & 332 & 61,88 \\
\hline Illes Balears & 93 & 42 & 63,37 \\
\hline La Rioja & 96 & 153 & 63,05 \\
\hline País Vasco & 100 & 540 & 69,51 \\
\hline Principado de Asturias & 90 & 42 & 45,59 \\
\hline Región de Murcia & 96,00 \\
\hline
\end{tabular}

Datos de Transparencia Internacional extraidos del ranking global del Índice de Transparencia de las Comunidades Autónomas, (Transparencia Internacional, 2014). 
ción de Transparencia Internacional y la media Meloda de los datos de cada comunidad autónoma $(0,6473)$, se puede observar que ésta es mayor que las anteriores y, por tanto, se puede identificar que hay una elevada correlación entre el Índice de Transparencia Internacional y la métrica Melo$d a$. Este resultado indica que las instituciones autonómicas que publican una mayor información y, por tanto, obtienen una mayor puntuación en el Índice de Transparencia Internacional, lo hacen en formato reutilizable, mientas que aquellas instituciones que publican poca información lo hacen en formatos inadecuados para su reutilización o esta información es de reutilización básica.

La totalidad de los datos abiertos de Aragón y Canarias son de reutilización avanzada

\section{Conclusiones}

El $88,24 \%$ de las comunidades autónomas (las excepciones son la Comunidad de Madrid y la Región de Murcia) ha implementado un portal de datos abiertos. El nivel de implementación que presentan estos portales es muy variable entre sí, ya que cada uno tiene publicados un número muy diferente de conjuntos de datos. El grado de adecuación para la reutilización de los conjuntos de datos también varía entre portales, siendo el 95,15\% de reutilización avanzada con alguna característica mejorable y de reutilización avanzada.

Atendiendo a la calificación obtenida por conjunto de datos, se puede identificar que las puntuaciones obtenidas son más bajas debido al grado de implementación de las variables información geolocalizada e información en tiempo real ya que la puntuación promedio obtenida en estas variables es de $49,16 \%$ y $29,13 \%$ respectivamente. Se observa que estas variables, dependiendo del tipo de conjunto de datos, podrían generar un mayor valor en el contexto de las ciudades inteligentes (Abella; Ortiz-de-Urbina-Criado; DePablos-Heredero, 2015a; 2015b). Las variables estándares técnicos, acceso, legal y modelo de datos tienen una media de $81,79 \%$, 91,82\%, 94,05\% y $91,35 \%$ respectivamente.

Un $89,28 \%$ de los datos abiertos publicados se ajustan a la definición de estándar abierto cumpliendo con los principios generales de la publicidad activa; es decir, se publican datos en formatos reutilizables facilitando su identificación y, en algunos casos, su localización (artículo 5 de la Ley 19/2013), garantizando el acceso a la información pública actualizada de manera sostenible a lo largo del tiempo. Además se cumple con la definición de estándar abierto como recoge en el anexo, letra k, la Ley 11/2007, de acceso electrónico de los ciudadanos a los servicios públicos (vigente hasta la entrada en vigor de la Ley 39/2015, de 1 de octubre) (España, 2007a).

Si se atiende a la interoperabilidad técnica, artículo 11 del $R D$ 4/2010, por el que se regula el Esquema nacional de interoperabilidad (España, 2010), y a los formatos puestos a disposición para su reutilización en los portales de datos abiertos, Ley 37/2007, sobre reutilización de la información del sector público (modificada por la Ley 18/2015) (España, 2007b), se observa que los conjuntos de datos publicados por comunidades autónomas como Aragón e Islas Canarias emplean estándares abiertos con metadatos como rdf, rss, json y xml.

El 91,45\% de los conjuntos de datos estudiados favorecen un uso no limitado o únicamente con autoría (CC BY 4.0). Atendiendo a la variable acceso a la información, el 65,22\% de los datos abiertos posibilita el acceso vía web con parámetros, mientras que el $32,19 \%$ tiene un acceso completo (API de datos). Se observa que la implementación de los datos abiertos por comunidades autónomas en España se encuentra en general en una fase madura. Las entidades que están liderando este proceso son Aragón, Catalunya, Navarra y País Vasco.

Como se identifica en Abella (2015), "existe una relación positiva entre el mecanismo de acceso y el incremento de uso de la información".

Además, como se ha identificado en la fase de análisis, existe una relación directa entre la puntuación de Transparencia Internacional y la media Meloda de los datos de cada comunidad, lo que supone una estrecha relación entre las instituciones autonómicas que publican una mayor cantidad de datos y el mayor nivel de reutilización de los mismos.

\section{Hay una elevada correlación entre el Índice de Transparencia Internacional y la métrica Meloda}

Respecto a la métrica Meloda, se ha identificado que la heterogeneidad de los portales de datos abiertos y sus conjuntos de datos hacen que el análisis del nivel de reutilización de los mismos resulte complejo y exigente. Esto se debe a que el nivel de cumplimiento de la normativa de referencia sobre reutilización y acceso a la información pública de aplicación en España es disperso y presenta carencias notables en cuanto a la completitud y actualización de los conjuntos de datos.

\subsection{Implicaciones para la gestión de la información}

Los resultados ponen de manifiesto algunas cuestiones directamente aplicables a la gestión de la información en las organizaciones públicas. Estos resultados tienen interés para todas las organizaciones de las comunidades autónomas que publican datos abiertos y otras administraciones públicas, así como para ciudadanos, reutilizadores del sector infomediario, social y académico, y también para usuarios profesionales. Es importante promover y ampliar una cultura de datos abiertos en España porque su penetración actualmente es baja. A partir del estudio Consumption of public institutions' open data by Spanish citizens (Gértrudix; Gertrudis-Casado; Álvarez-García, 2016) se identifica que:

- un $57 \%$ de las personas encuestadas mostró su desacuerdo respecto a que las instituciones públicas proporcionan los datos abiertos de manera accesible y fácil de encontrar; - un $50 \%$ opina que las instituciones públicas no facilitan el análisis y la interpretación de los datos abiertos mediante herramientas y aplicaciones sencillas. 
A partir de la identificación de la situación actual sobre reutilización de datos abiertos, se podrían articular estrategias de mejora de la publicación de la información basadas en los conjuntos de datos que presentan una reutilización inadecuada o básica. Las estrategias de mejora podrían basarse en una transparencia adaptable, mediante formatos y con contenidos adecuados y adaptados a cada perfil de usuario según la información de su interés, la publicación de datos abiertos bajo licencias abiertas estandarizadas (PDDL o ODbL) y en dirigir los procesos de gestión de la información hacia la apertura por defecto (Vicente-Paños; González-Ladrón-de-Guevara, 2017). Dichas estrategias de mejora requerirían de un cambio de orientación en las políticas de comunicación y dinamización (Gértrudix; Gertrudis-Casado; Álvarez-García, 2016), y permitirían avanzar en la consolidación de la rendición de cuentas, hacia el impulso de una gobernanza inteligente y la dinamización de la transformación digital y el crecimiento económico mediante datos abiertos.

\subsection{Limitaciones y futuras líneas de investigación}

Pese a la representatividad, la consistencia de la muestra seleccionada y la métrica utilizada, se evidencia el peso determinante del factor humano en el proceso de análisis de los conjuntos de datos por parte de los autores de este trabajo, es decir, con un proceso de medición sistematizado realizado mediante sistemas de medición informatizados se podrían haber corregido los posibles sesgos del análisis realizado.

Una futura línea de investigación debería considerar la limitación presentada. Debería ser capaz de estudiar en qué medida los indicadores de reutilización de la métrica $\mathrm{Me}$ loda se están utilizando para medir los conjuntos de datos disponibles por parte de los gestores de información de los portales de datos abiertos de las comunidades autónomas, para identificar si efectivamente estos indicadores han incrementado la reutilización de información pública.

Otra futura línea de investigación debería estudiar la relación existente entre el nivel de reutilización de los conjuntos de datos y la reutilización por parte de los ciudadanos y el sector infomediario de estos datos abiertos. Además, convendría conocer si los conjuntos de datos reutilizados son de las categorías donde el nivel de reutilización es mayor.

\section{Notas}

1. Transparencia activa: publicidad activa, obligación de difundir información pública (artículos 6, 7 y 8 de la Ley de Transparencia, acceso a la información pública y buen gobierno) sin esperar una solicitud concreta.

2. Transparencia pasiva: derecho de las personas a acceder a la información pública (artículo 12 de la Ley de Transparencia, acceso a la información pública y buen gobierno).

3. Transparencia colaborativa: datos en crudo (datos del gobierno abierto) publicados bajo estándares abiertos y reutilizables que posibiliten el derecho de la ciudadanía al acceso, redistribución y reutilización de los mismos (Anexo Ley 18/2015, de 9 de julio, por la que se modifica la Ley $37 / 2007$, de 16 de noviembre, sobre reutilización de la información del sector público).
4. Transparencia por diseño: predefinición de los procesos de gestión de la información en todos los niveles de la organización. Incluyendo (Vicente-Paños; Jordán-Alfonso, 2016; García-González, 2016):

- distribución de roles organizativos;

- predefinición del derecho de acceso a la información pública;

- creación de unidades de información (según el tamaño de la organización);

- codefinición con oficinas, unidades o servicios de la organización de un mapa de obligaciones en materia de transparencia;

- descripción de procesos mediante un modelo entidad-relación;

- integración de los sistemas de gestión de la información de la organización para cumplir con los principios generales de la publicidad activa;

- valoración de la idoneidad de implantar un sistema de gestión documental (SGD);

- creación del catálogo o repositorio de datos;

- publicación de datos abiertos;

- implantación de un sistema de solicitud de nuevos conjuntos de datos.

5. Transparencia abierta: en el contexto de este estudio se incorpora el matiz abierto a la transparencia, asumiendo que la transparencia debería implicar una publicación de la información pública en formatos abiertos. Esta propuesta estaría en la línea de la transparencia colaborativa introducida por otros autores de referencia (Villoria; Cruz-Rubio, 2015).

\section{Bibliografía}

Abella, Alberto (2015). "Costes del acceso a los repositorios de información". En: XIV Workshop Rebiun de proyectos digitales / VI Jornadas OS-Repositorios, marzo. https://goo.gl//cMWEI

Abella, Alberto (2016). "Full description of Meloda". Meloda. Portal for data publishers and profesional reusers of data. http://www.meloda.org/full-description-of-meloda

Abella, Alberto; Ortiz-de-Urbina-Criado, Marta; De-PablosHeredero, Carmen (2014). "Meloda, métrica para evaluar la reutilización de datos abiertos". El profesional de la información, v. 23, n. 6, pp. 582-588. https://doi.org/10.3145/epi.2014.nov.04

Abella, Alberto; Ortiz-de-Urbina-Criado, Marta; De-PablosHeredero, Carmen (2015a). "Information reuse in smart cities' ecosystems". El profesional de la información, v. 24, n. 6, pp. 838-844. https://doi.org/10.3145/epi.2015.nov.16

Abella, Alberto; Ortiz-de-Urbina-Criado, Marta; De-PablosHeredero, Carmen (2015b). "The ecosystem of services around smart cities: An exploratory analysis". En: Centeris. Conference on enterprise information systems, Vilamoura, Procedia computer science, v. 64C, pp. 1076-1081. https://doi.org/10.1016/j.procs.2015.08.554

Arenilla, Manuel (2014). "Nuevas formas de gestión basadas en las TIC". En: Arenilla, Manuel (coord). Administración 
2032. Teclas para transformar la Administración Pública española. Madrid: Instituto Nacional de Administración Pública, pp. 165-177. ISBN: 9788473513579.

Berners-Lee, Tim (2009). Linked data.

https://www.w3.org/Design/ssues/LinkedData.htm/

Carrara, Wendy; San-Chan, Wae; Fischer, Sander; VanSteenbergen, Eva (2015). Creating value through open data: Study on the impact of re-use of public data resources. European Union. ISBN: 9789279527913

https://doi.org/10.2759/328101

Comisión Europea (2003). “Directiva 2003/98/CE del Parlamento Europeo y del Consejo, de 17 de noviembre de 2003, relativa a la reutilización de la información del sector público". Diario oficial de la Unión Europea, 18 de noviembre. https://www.boe.es/buscar/doc. php?id=DOUE-L-2003-82244

Comisión Europea (2013). “Directiva 2013/37/UE del Parlamento Europeo y del Consejo, de 26 de junio de 2013, por la que se modifica la Directiva 2003/98/CE relativa a la reutilización de la información del sector publico". Diario oficial de la Unión Europea, 27 de junio.

https://www.boe.es/diario_boe/txt.php?id=DOUE-L-2013-81251

Datos.gob.es (2016). Iniciativa de datos abiertos del gobierno de España: impacto.

http://datos.gob.es

España (2007a). "Ley 11/2007, de 22 de junio, de acceso electrónico de los ciudadanos a los servicios públicos". BOE, n. 150, 23 de junio, pp. 27150-27166.

http://www.boe.es/diario_boe/txt.php?id=BOE-A-2007-12352

España (2007b). "Ley 37/2007, de 16 de noviembre, sobre reutilización de la información del sector público". BOE, n. 276, 17 de noviembre, pp. 47160-47165.

https://www.boe.es/diario_boe/txt.php?id=BOE-A-2007-19814

España (2010). "Real decreto 4/2010, de 8 de enero, por el que se regula el esquema nacional de interoperabilidad en el ámbito de la administración electrónica". BOE, n. 25, 29 de enero, pp. 8139-8156.

http://www.boe.es/buscar/doc.php?id=BOE-A-2010-1331

España (2013). "Ley 19/2013, de 9 de diciembre, de transparencia, acceso a la información pública y buen gobierno". $B O E$, n. 295, 10 de diciembre, pp. 97922-97952.

https://www.boe.es/buscar/doc.php?id=BOE-A-2013-12887

España (2015). "Ley 18/2015, de 9 de julio, por la que se modifica la Ley $37 / 2007$, de 16 de noviembre, sobre reutilización de la información del sector público". BOE, n. 164, 10 de julio, pp. 57436-57450.

https://www.boe.es/diario_boe/txt.php?id=BOE-A-2015-7731

European Data Portal (2017). “Open data in Europe”. European Data Portal.

https://www.europeandataportal.eu/en/dashboard

Ferrer-Sapena, Antonia; Peset, Fernanda; AleixandreBenavent, Rafael (2011). "Acceso a los datos públicos y su reutilización: open data y open government". El profesional de la información, v. 20, n. 3, pp. 259-268. https://doi.org/10.3145/epi.2011.may.03
Ferrer-Sapena, Antonia; Sánchez-Pérez, Enrique A. (2013). "Open data, big data: ¿hacia dónde nos dirigimos?". Anuario ThinkEPI, v. 7, pp. 150-156.

http://eprints.rclis.org/21006/1/150-156-Ferrer-SanchezOpen-data-Big-data.pdf

García-González, María (2016). “Administración electrónica: por qué implantar una política de gestión de procesos institucionales en las administraciones públicas". El profesional de la información, v. 25, n. 3, pp. 473-483. https://doi.org/10.3145/epi.2016.may.17

Gértrudix, Manuel; Gertrudis-Casado, María-Carmen; Álvarez-García, Sergio (2016). "Consumption of public institutions' open data by Spanish citizens". El profesional de la información, v. 25, n. 4, pp. 535-544.

https://doi.org/10.3145/epi.2016.jul.03

Pipino, Leo L.; Lee, Yang W.; Wang, Richard Y. (2002). "Data quality assessment". Communications of the ACM, v. 45, n. 4, pp. 211-218.

https://goo.gl/hE1JK8

Ramió, Carles (2010). "Teoría de la organización y administración pública". En: Ramió, Carles. Teoría de la organización y administración pública. Barcelona: Tecnos, pp. 17-41. ISBN: 8430934502 https://goo.gl/et8ayh

Ren, Guang-Jie; Glissmann, Susanne (2012). "Identifying information assets for open data. The role of business architecture and information quality". En: $7^{\text {th }}$ IEEE Int/ conf on e-commerce technology (CEC'05), Sept., pp. 94-100. http://doi.ieeecomputersociety.org/10.1109/CEC.2012.23

Transparencia Internacional España (2016). Índice de las Comunidades Autónomas (Incau).

http://transparencia.org.es/incau-2016

Transparencia Internacional España (2014). Ranking global, Índice de Transparencia de las Comunidades Autónomas (Incau).

http://webantigua.transparencia.org.es/incau_2014/ ranking_global_incau_2014.pdf

Ubaldi, Barbara (2013). "Open government data: Towards empirical analysis of open government data initiatives". OECD Working papers on public governance, 22. OECD Publishing.

http://dx.doi.org/10.1787/5k46bj4f03s7-en

Vicente-Paños, Adrián (2015). “De la gestión de la información... hacia ¿el open data?". En: Cotino-Hueso, Lorenzo; Sahuquillo-Orozco, José-Luis; Corredoira-Alfonso, Loreto (eds.). El paradigma del gobierno abierto. Retos y oportunidades de la participación, transparencia y colaboración. Madrid: Universidad Complutense de Madrid, pp. 313-330. ISBN: 978846069678078. http://eprints.ucm.es/35859

Vicente-Paños, Adrián (2016). "Gestión de la innovación en organizaciones públicas: el open data en el frontispicio del open government". En: Balcells-Padullés, Joan; Borge-Bravo, Rosa; Delgado-García, Ana-María; Fiori, Mirela; Julià-Barceló, Maria; Marsan-Raventós, Clara; Peña-López, Ismael; 
Pifarré-de-Moner, María-José; Torrubia-Chalmeta, Blanca; Vilasau-Solana, Mònica (coord.). Building a European digital space: Actas del 12ㅇ Congreso intl internet, derecho y política. Universitat Oberta de Catalunya, Barcelona, 7-8 de julio de 2016, pp. 418-440. ISBN: 9788460896708

https://goo.gl/ZVRTrs

Vicente-Paños, Adrián; Gónzalez-Ladrón-de-Guevara, Fernado (2017). Nuevas tendencias en estrategia de servicio en el sector público. NovaGob Estudios, n. 5. NovaGob; Universitat Politécnica de Valencia.

https://goo.gl/BSVBrF

Vicente-Paños, Adrián; Jordán-Alfonso, Aurea (2016).
“Open data, acceso a la información pública y su reutilización: análisis y propuesta de mejora de la situación de la transparencia en la Comunidad Valenciana". En: III Congreso internacional del avance del gobierno abierto / I Congreso de buen gobierno y transparencia de la Comunitat Valenciana, Septiembre.

https://www.ivoox.com/12961905

Villoria, Manuel; Cruz-Rubio, César (2015). "Govern obert, transparència i rendició de comptes: marc conceptual". En: Villoria, Manuel (dir.); Forcadell, Xavier (coord.). Bon govern, transparència i integritat institucional al govern local. Barcelona: Diputació de Barcelona, pp. 61-89. ISBN: 978 8498037135

\section{No descuides mantener actualizados tus datos en el Directorio EXIT} (EXpertos en el Tratamiento de la Información)

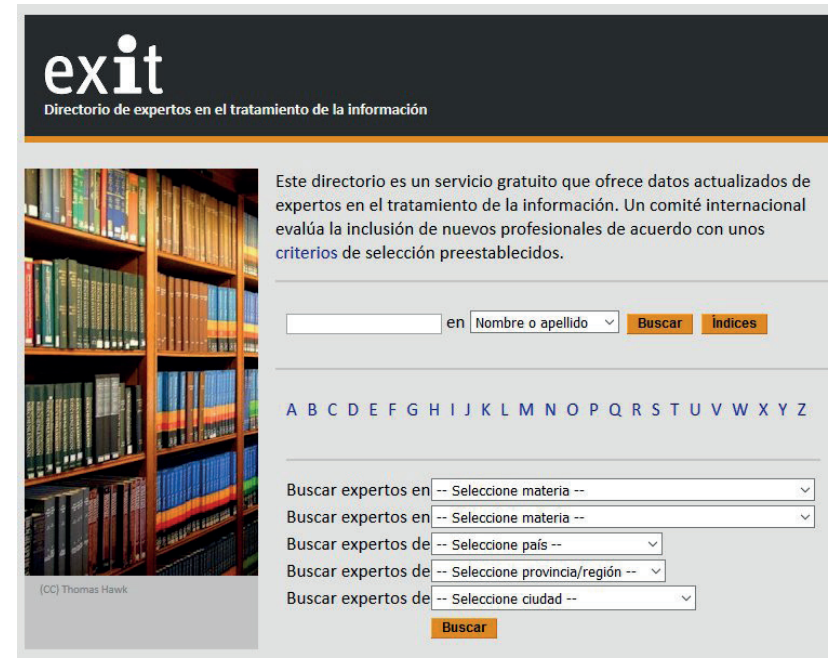

Puedes incluir enlaces a tus páginas de las redes sociales

Especialidades más representadas en EXIT $\quad \mathbf{n}^{\circ}$

Gestión de la información y del conocimiento 987

Web 2.0 y redes sociales 844

Biblioteca universitaria $\quad 762$

Recuperación de información y búsquedas 655

Información científico-técnica 632

Biblioteca digital 551

Planificación y gestión 500

Bibl. especializada / Centro de documentación 446

Análisis y diseño de sistemas de información 447

Gestión de contenidos $\quad 446$

Open access 447

Comunicación 569

Catalogación, clasificación e indexación $\quad 419$

Bases de datos (contenidos) 394

Educación, formación, alfin 433

Revistas electrónicas 343

Bibliometría y cibermetría 385

Marketing y promoción 354

Documentación en general 327

Arquitectura de la información 321

Servicios de internet en general 294

Información biomédica 305

Diseño de webs 296

Usabilidad, interfaces e interacción 279

Biblioteca pública $\quad 267$

Software documental 244

Ontologías, metadatos, taxonomías y tesauros 236

Periodismo 310

Multimedia y documentación audiovisual 253

Información humanística 246

http://directorioexit.info 